\title{
U-model enhanced dynamic control of a heavy oil pyrolysis/cracking furnace
}

Q.M. Zhu\#1, 2, D.Y. Zhao*\#2, Shuzhan Zhang\#2, Pritesh Narayan\#1

\author{
\#1 Department of Engineering Design and Mathematics \\ University of the West of England \\ Frenchy Campus Coldharbour Lane \\ Bristol, BS16 1QY, UK \\ Email: quan.zhu@uwe.ac.uk \\ Email: pritesh.narayan@uwe.ac.uk
}

\author{
\#2 Department of Chemical Industrial Equipment and Control Engineering \\ College of Chemical Engineering \\ China University of Petroleum \\ Qingdao, 266580, China \\ Email: dyzhao@upc.edu.cn \\ Email: sz_8866@163.com \\ * Corresponding author
}

\begin{abstract}
This paper proposes a case study in the control of a heavy oil pyrolysis/cracking furnace with a newly extended U-Model based Pole Placement Controller (U-PPC). The major work of the paper includes 1) establishing a control oriented nonlinear dynamic model with Naphtha cracking and thermal dynamics, 2) analysing a U-model (i.e. control oriented prototype) representation of various popular process model sets, 3) designing the new U-PPC to enhance the control performance in pole placement and stabilisation, 4) taking computational bench tests to demonstrate the control system design and performance with a user-friendly step by step procedure.
\end{abstract}

Keywords: Heavy oil cracking furnace, Kumar molecular dynamics model for Naphtha cracking, U-model, Umodel enhanced controller design, pole placement controller, stabilisation controller, and computational experiments.

\section{Introduction}

The introduction consists of five sub-sections: a review of existing representative approaches in modelling of oil cracking furnaces, a review of control approaches to such furnace operations, a brief overview of U-model based control system design and, with reference to the critical survey/analysis, justifying the necessity of the study and listing the major contributions of the current work, and finally summarising the remaining sections of the study.

\subsection{Modelling of heavy oil cracking furnace}

Cracking feedstock, heated and conveyed into the furnace tube of the radiant section of a furnace, absorbs heat from the external radiation chamber, causing heating and cracking. In order to obtain satisfactory quality and quantity of product, it is necessary to build up reference models to describe the reaction in the tube, and the heat transfer dynamic process both inside and outside the tube. This involves many variables for controlled operation, such as length of material temperature, pressure distribution and product distribution. . It has been noted (Zheng, Zhang, Qi, and Zhou 2015) that smoke flow from the radiation interior is subject to intense turbulence, and furnace temperature distribution varies with burner positioning and actual combustion conditions. In regards to modelling, zero dimensional models cannot properly reflect temperature distribution inside the furnace hearth, and high dimensional models of flue gas temperature distribution inside the furnace inevitably lead to complexity in analysis and computation (particularly for online control). Therefore, choice of the model in the heat transfer in radiation chamber must compromise between accuracy and complexity.

A brief summary of existing modelling work is given here. Representatively there have been heat transfer models in tube furnace (Guo 1981, Hasan, Perera, Baddage, and Persson 2012), one dimensional temperature models for gradient furnace (Guo, 1981), two dimensional models (Rainara, Fleliers, and Froment 1988), and high dimensional models (Cheema, Ali, and Park 2016).

For model analysis and simulation, there has been examination of pure radiation heat transfer in the cylindrical tubular heater (Yang, Huang, and Yang 1989), studies on radial temperature distribution of the furnace tube (Heynderickx, Cornelis, and Froment 1992), and simulation of radiation section of cracking furnaces (Fleliers 
1990). Even though these models cover dynamics, they have seldom considered nonlinear effects existing in the process.

\subsection{Control of heavy oil cracking furnace}

The advanced control and optimisation of ethylene production processes has seen much theoretical/analytical development and application. For example, optimization and advanced control of thermal cracking processes (Savu 2010), operation optimisation of naphtha cracking furnaces (Huang 1980; Yu, Wang, and Wang 2015), closed-loop steady-state real-time optimisation systems (CTRTO) implemented by the Mobil Chemical Company of the State of Texas (Apostolos 1998), which demonstrated application of computer control to the Mobil ethylene plant.

The most successful method of advanced process control technology used in the process of ethylene production has been the dynamic model control (DMC) method (Shi, Su, Cao, Li, Liang, and Zhong 2015), represented by the Plus DMC of the AspenTech Company. In 1997 it was reported that Plus DMC accounted for 70\% of worldwide ethylene plant advanced process control (APC). Plus DMC mainly consists of estimating, linear programming, and dynamic control modules (Li, Li, Lei, Chen, Ren, and Cao 2011). The Setpoint Company (1990) has developed a computer optimization control system for an olefin plant, which has been used for stable control, constrained control, local optimization control, overall optimization control of four-level hierarchical control strategy, and it has achieved good results. Additionly, ABB Simcom company has developed an olefin plant simulation and optimization software package OPSO (Olefins Plant Simulation and Optimization), which has been successfully used in many ethylene plants ( $\mathrm{Li}, \mathrm{Li}$, Lei, Chen, Ren, and Cao 2011).

It has been observed that the most control strategies used so far have been PID or PID type controllers, and nonlinear process control is still an area to be explored. Generally applicable controller designs may therefore be developed, exploiting ever faster and more flexible computation resources.

\subsection{U-model enhanced control system design}

Since the proposition of U-model (in terms of "providing concise and applicable control oriented model structure for designing complex nonlinear control systems") in the first author's PhD thesis (Zhu 1989), a series of the corresponding U-model enhanced control design approaches have been developed (Zhu, Zhao and Zhang 2016). It has been claimed that the U-model based control system design methodology radically reduces complexity in classical control system design and plant model based design. The role of the plant model is effectively reduced to that of a reference for converting to the U-model domain and determining the next-step controller output by resolving one of the U-model roots (Zhu, Zhao and Zhang 2016). U-model based control is based on the hypothesis that it is possible to use linear methodologies to directly provide solutions for control of a large class of smooth nonlinear dynamic plant models, and thus, by principle of parsimony, simplify and generalise nonlinear system control design. The whole framework for U-model based control system design is shown in Figure 1.

The U-model systematically transforms smooth (polynomial) nonlinear models into a class of plant input/controller output $u$ based polynomials, thus resolving plant dynamic inversion, by finding one of the roots of the U-model. The advantage of the control oriented model transform is to simplify controller design procedures in line with those for linear control system design. It should be mentioned that under such a transform there is nothing lost from original nonlinear models. Regarding U-model based control research development, Zhu and Guo (2002) established the foundation framework, with pole placement control of NARMAX (Nonlinear AutoRegressive Moving Average with eXogenous input) models. More recently, U-model enhanced control system design has been expanded to U-General Predictive Control (UGPC) (Du, Wu, and Zhu 2012) and U-Sliding Mode Control (USMC) (Zhu, Zhao, and Zhang 2016). For nonlinear state space models, a UBackstepping framework is under development to iteratively "step backward" to determine control output in resolving multi loop U-models. In brief, U-model covers a wide range of nonlinear plants, which may be used as a basis for applying linear control techniques. This should be considered with another potential research topic: expanded alternative U-models for neural networks, network control modes, multi-agents, etc. This may permit reducing the complexity of the current model structures of some newly developed representative control system design approaches (Shi, Li, Wu, and Lim 2016, Shao, Miao, and Zhang 2015, Xing, Zhong, and Li 2015). It should be noted that U-model based approaches maintain the same control performance as other approaches, but make their design procedure more feasible, concise, and general, without unnecessary design repetition on plant model changes. Even for linear plant models, U-model approaches have the same merits over their classical counterparts. 


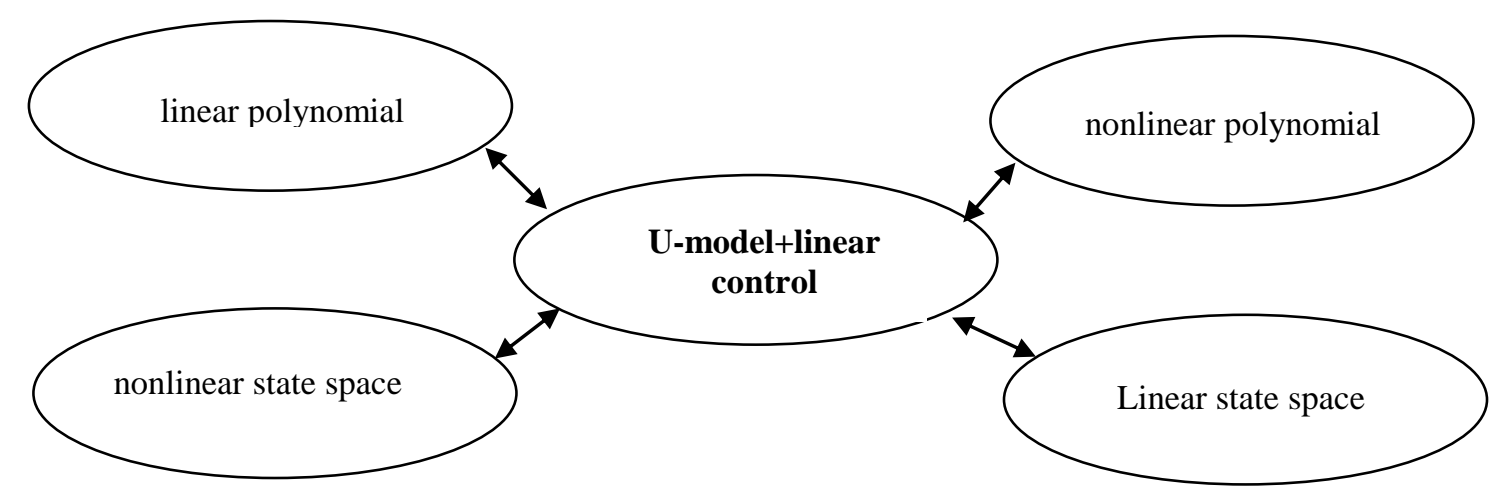

Figure 1 U-model based control system design framework

\subsection{Justification and contribution of the study}

The study mainly considers establishing a systematic procedure in designing nonlinear dynamic control systems for nonlinear dynamics in actual existing chemical engineering processes which are seldom considered in modelling and control due to difficulty and lack of generality in analysis, design, and computation.

The design procedure includes, 1) modelling, from a principle model, and formulisation of a control oriented model to U-model, 2) control system design coping with nonlinearities and a conditional stable dynamic process, and 3) a bench test example. It should be noted that the control system design approach developed in the study is fundamentally different from a Jacobian linearization for small range operation, which is the base for almost all other linear control system design approaches. Furthermore, this is the first study of issues associated with control of conditionally stable nonlinear process within the U-model framework, which has not theoretically proved the controller, but heuristically formulated and numerically demonstrated with the bench test example.

\subsection{Section organisation of the study}

The rest of the paper is organised into the following sections. In section 2, a nonlinear dynamic model is built up for heavy oil pyrolysis/cracking furnace, which is the basis for the process temperature control. In section 3 , the $\mathrm{U}$-model is introduced and the associated properties are presented to provide a proper description of the model structure and characteristics. Its relation to Hammerstein, Wiener, and NARMAX models are explained to show the generality and applicability in control system design. Subsequently, remarks with examples are presented for explanation and understanding of the conversion from classical polynomials to the U-model set. A general Umodel based controller design procedure is explained with three different types of example. In section 4, The Umodel based temperature control of the heavy oil cracking furnace is designed in terms of pole placement and stabilisation. In section 5, a series of computational experiments are conducted to numerically demonstrate the theoretically developed results. In section 6 , a brief conclusion is drawn to summarise the results and to explain some potential research issues.

\section{Modelling of heavy oil cracking furnace}

It must be acknowledged at the outset that the modelling description and re-organisation are largely resourced from a $\mathrm{PhD}$ thesis (Hu 2005) to enable formatting of a proper control oriented nonlinear dynamic model.

In common with many chemical engineering processes, proper temperature control is a requirement for the quality and quantity of chemical products. The pyrolysis process for heavy oil is very much dependent on the temperature in the furnace and the outer wall of the tube. The steady-state model of the pyrolysis process, linking the temperature of the outer wall of the tube/output to the temperature in the furnace/input, is the basis for expansion into the corresponding dynamic model for temperature control. In general, the steady-state model of a cracking furnace includes three balance principles, material balance (cracking reactions in chemical kinetics models), heat balance and momentum balance. Similar to finite element analysis, Kumar molecular dynamics model structure (Kumar and Kunzru 1985) can be used to describe the input and output relationships in terms of dynamics and steady states on a small section of the furnace, and thus the furnace characteristics can be extrapolated.

With reference to the detailed modelling work (Hu 2005), the dynamic variation of furnace wall temperature can be determined by the following differential equation 


$$
\frac{d T_{W}\left(t_{c}\right)}{d t_{c}}=\pi D_{0} \frac{C_{b} H_{s}\left[\left(\frac{T_{G}\left(t_{c}-1\right)}{100}\right)^{3}-\left(\frac{T_{W}\left(t_{c}-1\right)}{100}\right)^{3}\right]-k\left(T_{W}\left(t_{c}-1\right)-T\right)}{G_{W} \cdot C_{P W}}
$$

where for a particular model representing high temperature range of a cracking furnace.

$t_{c}$ is the continuous time.

$T_{W}$ is the temperature of the outer wall of the tube (the flue gas through radiation and convection to the outer wall of the furnace tube) and defined as the model output.

$T_{G}$ is the temperature in furnace and defined as the model input or control variable to regulate the temperature of the outer wall of the tube.

$C_{b} H_{s}$, the product, is absolute black body radiation constant $5.67 \mathrm{~W} /\left(\mathrm{m}^{2} \cdot \mathrm{K}^{3}\right)$

$D_{0}$ is external diameter of reaction tube $0.135 \mathrm{~m}$.

$T$ is the average temperature of pyrolysis gas, the average temperature of pyrolysis gas $1109 \mathrm{~K}$ (the high temperature range of cracking furnace: $973 \sim 1173 \mathrm{~K})$.

$k$ is the total heat transfer coefficient $6.463 \mathrm{~W} /\left(\mathrm{m}^{2} \cdot \mathrm{K}^{3}\right)$

$G_{W}$ is quality of unit length furnace tube $69 \mathrm{~kg} / \mathrm{m}$ (the quality range of high temperature section of cracking furnace: $67.2 \sim 69.9 \mathrm{~kg} / \mathrm{m}$ ).

$C_{P W}$ is the specific heat capacity of furnace tube material $822.6 \mathrm{~J} /(\mathrm{kg} \cdot \mathrm{K})$ (the specific heat capacity range in high temperature of cracking furnace: $758.35 \sim 873.56 \mathrm{~J} /(\mathrm{kg} \cdot \mathrm{K})$ ).

Remark 1: Equation (2.1) is also called as input and output dynamic model. In regard to its structure, it is a nonlinear continuous time model.

To facilitate digital controller design and implementation by computer or digital control instruments, discretise the continuous time model (2.1) into a discrete time model by a difference operator of

$$
\frac{d T_{W}\left(t_{c}\right)}{d t_{c}}=\frac{T_{W}(t)-T_{W}(t-1)}{\Delta t_{c}}
$$

where $t=1,2 \cdots$ is the sampling instance and $\Delta t_{c}$ is the sampling interval. Accordingly, the discrete time model is determined with

$$
T_{W}(t)=\theta_{1} T_{W}(t-1)+\theta_{2} T_{W}^{3}(t-1)+\theta_{3} T_{G}^{3}(t-1)+\theta_{4}(2.3)
$$

With reference to the given furnace model parameters above and setting the sampling interval $\Delta t_{c}=100$, it gives the parameter vector in (2.3)

$$
\begin{gathered}
\theta_{1}=1-\frac{\Delta t_{c} \pi D_{0} k}{G_{W} C_{P W}}=1-0.4645=0.95 \\
\theta_{2}=-\frac{\Delta t_{c} \pi D_{0} C_{b} H_{s}}{G_{W} C_{P W}(100)^{4}}=-0.00408 \\
\theta_{3}=\frac{{ }_{s} \Delta t_{c} \pi D_{0} C_{b} H_{s}}{G_{W} C_{P W}(100)^{4}}=0.00408 \\
\theta_{4}=\frac{\Delta t_{c} \pi D_{0} T}{G_{W} C_{P W}}=0.79
\end{gathered}
$$

$\left[\begin{array}{llll}\theta_{1} & \theta_{2} & \theta_{3} & \theta_{4}\end{array}\right]=\left[\begin{array}{llll}0.95 & -0.00408 & 0.00408 & 0.75\end{array}\right](2.5)$ 


\section{$3 \mathrm{U}$ model based control systems design}

This section presents the definition and foundation of the U-model, explains its representation of Hammerstein models, Wiener models, and NAMARX (Non-linear AutoRegressive Moving Average with eXogenous input) models, which have been widely used in process industries. With this control oriented model, it outlines the generic controller design procedure with a few of examples pole placement control, general predictive control, and a newly proposed stabilisation control.

\subsection{Definition of U-model}

Consider a general smooth nonlinear discrete time dynamic process/process

$$
y(t)=f(y(t-1), \ldots, y(t-n), u(t-1), \ldots, u(t-n))(3.1)
$$

where $y(t) \in R$ and $u(t-1) \in R$ are the output and input (also known as controller output in control system design) signals of the process respectively at discrete time instant $t(1,2, \ldots), n$ is the process order, and $f(\cdot)$ is a smooth linear or non-linear function. Then the process can be represented with a U-model structure which is defined as a controller output $u(t-1)$ oriented polynomial below (Zhu, Zhao and Zhang 2016),

$$
y(t)=\sum_{j=0}^{M} \lambda_{j}(t) u^{j}(t-1)
$$

where $M$ is the degree of model input (controller output) $u(t-1)$, the time varying parameter vector $\lambda(t)=\left[\begin{array}{lll}\lambda_{0}(t) & \cdots & \lambda_{M}(t)\end{array}\right] \in R^{M+1}$ is a function of past inputs and outputs $(u(t-2), \ldots, u(t-n), y(t-1), \ldots, y(t-n))$, and the other none $u(t-1)$ terms/parameters.

Property 1: Let $\varphi: \quad R^{L+1} \rightarrow R^{M+1}$ be the map from polynomial model (3.1) to U-model (3.2) and its inverse be $\varphi^{-1}$, that is $f\left(p_{i}, \theta_{i}\right) \stackrel{\varphi}{\longrightarrow} f\left(u^{j}, \lambda_{j}\right)$. Then it has the following properties (Zhu, Zhao, Zhang 2016)

1) The map is injective (one to one)

2) The map is surjective (onto)

3) Therefore, the map is bijective as it is both injective and surjective.

4) The map is invertible.

5) The map does not change any both model characteristics, such as output response, stability, dynamics and statics.

Remark 2: In consideration of the U-model (3.2), a control oriented prototype, there is no change of properties compared with various classical polynomial models in representation of (3.1) such as dynamic, stability, and input and output external relationship. Therefore, in model properties, U-model and classical polynomial models are equivalent. In the model expression, U-model is time varying in parameters (by re-organisation of classical model structure) and convenient for control systems design. In another point of view, U-model bridges nonlinear problems with linear solutions.

\subsection{Representations of $U$-model to the other classical model sets}

It has been studied that the U-model has solid base to cover various existing nonlinear polynomial models as its subsets. Therefore, those developed U-model based design procedures should be applicable to the model sets and their variations.

Examples of popular and widely recognised model sets are described here, to show the U-model potential coverage of application.

\section{Hammerstein models}

The basic Hammerstein model is a cascade structure of the nonlinear static (memoryless) block and the linear dynamic block which can be described by the following formulas (Babik and Dostal 2012) 


$$
\begin{aligned}
& X_{H}(t-1)=f_{H}(u(t-1)) \\
& y(t)=\sum_{i=0}^{m} b_{i}(t) X_{H}(t-1-i)-\sum_{i=1}^{n} a_{i}(t) y(t-i)
\end{aligned}
$$

where $y(t)$ and $u(t-1)$ are the output and input signals of the process respectively at discrete time instant $t(1$, $2, \ldots), X_{H}(t)$ is the output signal of the nonlinear static block, and $f_{H}($.) is a non-linear function of input $u(t-1)$.

The corresponding equivalent U-model can be expressed as

$$
\begin{aligned}
& y(t)=\lambda_{0}(t)+\lambda_{1}(t) X_{H}(t-1) \\
& =\lambda_{0}(t)+\lambda_{1}(t) f_{H}(u(t-1))
\end{aligned}
$$

where

$$
\begin{aligned}
& \lambda_{0}(t)=\sum_{i=1}^{m} b_{i}(t) X_{H}(t-1-i)-\sum_{i=1}^{n} a_{i}(t) \mathrm{y}(t-i) \\
& \lambda_{1}(t)=b_{0}
\end{aligned}
$$

Remark 3: Hammerstein models can be used to describe many different processes, especially if their main nonlinear behaviour is induced by actuators such as valves, an essential component for controlling of fluid in flow and pressure in many chemical engineering applications (Huang and Wang 2012). It has been observed (Zhang, Li, and Chen 2008) that this model structure has been successfully applied to chemical processes such as heat exchang, distillation, and biological processes.

\section{Wiener models}

The basic Wiener model is a cascade structure of the linear dynamic block and the nonlinear static block which can be described by the following formulas (Babik and Dostal 2012).

$$
\begin{aligned}
& X_{W}(t)=\sum_{i=0}^{m} b_{i}(t) u(t-1-i)-\sum_{i=1}^{n} a_{i}(t) X_{W}(t-i) \\
& y(t)=f_{W}\left(X_{W}(t)\right)
\end{aligned}
$$

where $y(t)$ and $u(t-1)$ are the output and input signals of the process respectively at discrete time instant $t(1$, $2, \ldots), X_{W}(t)$ is the output signal of the linear dynamic, and $f_{W}($.$) is a non-linear function of X_{W}(t)$.

The corresponding equivalent U-model can be expressed as

$$
\begin{aligned}
& X_{W}(t)=\lambda_{0}(t)+\lambda_{1} u(t-1) \\
& y(t)=f_{W}\left(X_{W}(t)\right)
\end{aligned}
$$

where

$$
\begin{aligned}
& \lambda_{0}(t)=\sum_{i=1}^{m} b_{i}(t) X_{H}(t-1-i)-\sum_{i=1}^{n} a_{i}(t) y(t-i) \\
& \lambda_{1}(t)=b_{0}
\end{aligned}
$$

Remark 4: Wiener models have been used to describe the control of pH neutralisation processes (Lee, Park, Lee, and Lee 2009) and solid oxide fuel cell systems (Mahmoodia, Poshtana, Jahed-Motlaghb, and Montazeria 2009). 
NAMAX models

The general NARMAX polynomial model (Billings 2013) can be expressed as

$y(t)=\sum_{l=0}^{L} p_{l}(t) \theta_{l}(3.9)$

where the regression terms $p_{l}(t)$ are the products of past inputs and outputs such as $u(t-1) y(t-3)$, $u(t-1) u(t-2), y^{2}(t-1)$, and $\theta_{l}$ are the associated parameters.

The corresponding equivalent U-model can be expressed as

$y(t)=\sum_{j=0}^{M} \lambda_{j}(t) u^{j}(t-1)$

Such an example ((Zhu, Zhao and Zhang 2016)), from polynomial model to the U-model conversion, is shown below.

The polynomial model is

$y(t)=0.1 y(t-1) y(t-2)-0.5 y(t-1) u^{2}(t-1)+0.8 u(t-1) u(t-2)$

And the U-model can be determined in notation of (3.2)

$y(t)=\lambda_{0}(t)+\lambda_{1}(t) u(t-1)+\lambda_{2}(t) u^{2}(t-1)$

where $\lambda_{0}(t)=0.1 y(t-1) y(t-2), \lambda_{1}(t)=0.8 u(t-2)$, and $\lambda_{2}(t)=-0.5 y(t-1)$.

Remark 5: NAMAX models can be used to represent wide range of smooth nonlinear dynamic systems and good approximation to hard nonlinear dynamic systems as well (Billings 2013). It should be noted that even NARMAX models have been well studied in the domain of system identification, it has almost not been systematically study in control system design due to the model structure not being a class of control oriented prototype.

\subsection{U-model enhanced controller design}

Assumption: The general process of (3.1) is assumed to be open loop stable and minimum phase (otherwise, need stabilisation means before design). Accordingly, signals within the designed closed loop are bounded.

In order to use linear polynomial mode based design approaches, define the desired process output as $y_{d}(t)$, which is specified either by designers or customers in advance. Accordingly, the relationship between a specified process output $y_{d}(t)$ and the requested corresponding controller output $u(t-1)$ can be expressed in terms of Umodel

$y_{d}(t)=\sum_{j=0}^{M} \lambda_{j}(t) u^{j}(t-1)$

This prototype establishes a framework for proposing a two-step design procedure.

Step 1 (design desired output $y_{d}(t)$ ): The first task of the design is to determine the desired process output $y_{d}(t)$ according to a specified performance index, here three typical examples listed for reference,

1) Pole Placement Control (PPC) (Zhu and Guo 2002) has been designed in terms of

$R y_{d}(t)=T w(t)-S y(t)$ 
where $y(t), y_{d}(t)$ and $w(t)$ are the process output, desired/designed process output, and reference input respectively. The polynomials $R, S$, and $T$ are used to specify the desired process output $y_{d}(t)$.

General Predictive Control (GPC) (Du, Wu, and Zhu 2012) has been designed in terms of

$$
J_{p}=E\left[(Y-W)^{T} Q_{1}(Y-W)+\Delta Y_{d}^{T} Q_{2} \Delta Y_{d}\right]
$$

where $Y, Y_{d}(t)$ and $W(t)$ are the vectors of $y(t), y_{d}(t)$ and $w(t)$ respectively. $Q_{1}$ and $Q_{2}$ are the corresponding weighting coefficient matrices.

It should be noted that the first step design for both controllers above do not require process model, which means once off design can be applicable to many different process models.

3) Stabiliser (newly presented in the study)

$y_{d}(t)=$ critical nonlinearity cancellation + linear compensation $(3.16)$

It should be noted that the design of critical nonlinearity cancellation and linear compensation can be separately achieved. Once again the design of linear compensation above does not require process model.

Step 2 (work out controller output $u(t-1)$ ): Then the remaining design task is to resolve one of the roots of (3.13) to obtain the controller output $u(t-1)$. That is

$u(t-1)=\Psi\left[y_{d}(t)-\sum_{j=0}^{M} \lambda_{j}(t) u^{j}(t-1)=0\right]$

where $\Psi[*]$ is a root-solving algorithm, such as Newton-Raphson algorithm. A detailed analysis on the root solving issues has been presented (Zhu and Guo 2002). As the second stage is a process model dependent process, each time of a plant model changed, its corresponding U-model need to be re-structured.

Remark 6: Regarding feasibility of the controller implementation and computational efficiency, as the controller is a once off design, it can be flexibly implemented with dedicated hardware. While a process model may change, (i.e. U-model change), additional implementation merely entails re-setting of the root solver. Thus, from hardware and software implementation, U-model based control systems are more economic, effective, and efficient. In computation, resolving inversion of the plant model is much simpler and quicker than resolving inversion of some format of the controller model and process model together. This is particularly critical in situations with limited power resources, such as control of Unmanned Aerial Vehicles (UAV).

\section{Control system design of heavy oil cracking furnace}

A typical cracking furnace control system is shown in Figure 2. There are three variables contributing to the temperature variation on the outer wall of the reaction tube: 1) the boiler feed water temperature, 2) the steam drum temperature, and 3) the fuel gas burn generated temperature through radiation and convection to the outer wall of the tube. In this study, the third is selected to be variable as the temperature control, defined as $T_{G}$ in (2.1), the other two assumed to be constants. In control system structure, the three variable controlled and tailored single variable controlled closed loop block diagrams are shown in Figure 3 (a) is the three variable controlled system (b) is the single variable controlled system. 


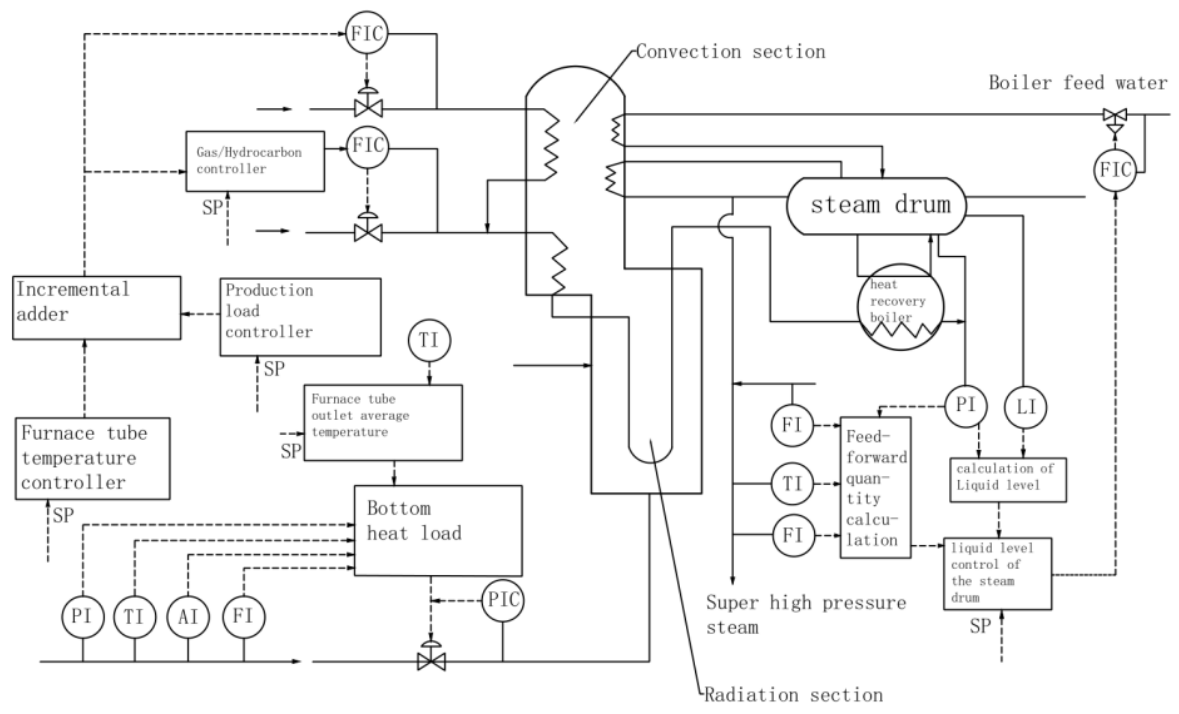

Figure 2 Cracking furnace control system

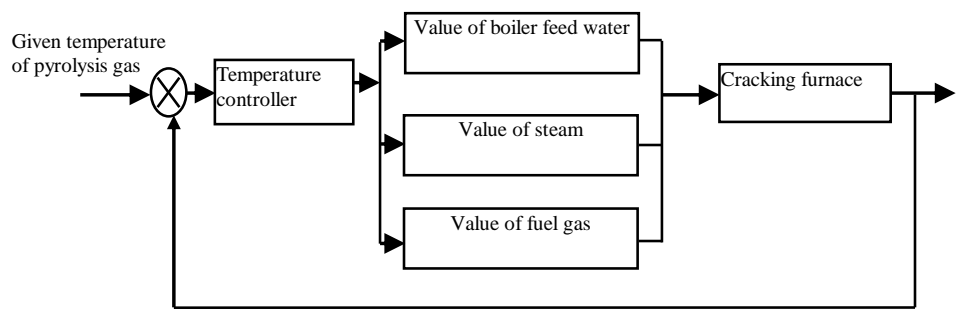

(a)

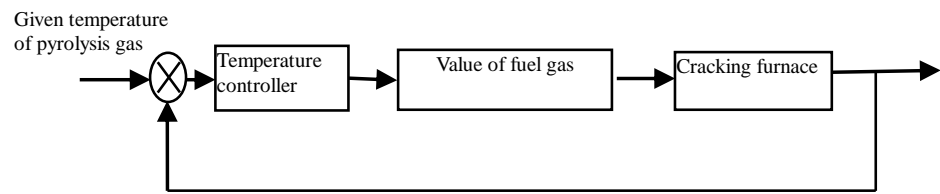

(b)

Figure 3 Control system block diagrams

For convenience, the notations in the original furnace wall model (2.3) are changed to use conventional notations in control system design

$y(t)=\theta_{1} y(t-1)+\theta_{2} y^{3}(t-1)+\theta_{3} u^{3}(t-1)+\theta_{4}(4.1)$

where $y(t)=T_{W}(t) \quad u(t)=T_{G}(t-1)$.

Accordingly, the corresponding U-model (4.1) can be expressed by 
$y(t)=\lambda_{0}(t)+\lambda_{3} u^{3}(t-1)$

where $\lambda_{0}(t)=\theta_{1} y(t-1)+\theta_{2} y^{3}(t-1)+\theta_{4} \quad \lambda_{3}(t)=\theta_{3}$

\subsection{Design of U-model based pole placement controller (U-PPC)}

The main feature of the pole placement control is to assign the closed loop nonlinear control system as behaving like a liner system with specified pole and steady errors. Here the U-PPC design procedure is specified for designing the control of the temperature on the outer wall of the reaction tube in the heavy oil pyrolysis/cracking furnace.

The controller structure in this study, as described with (3.14), is specified with,

$R y_{d}(t)=T w(t)-S y(t)(4.3)$

where the three polynomials are specified to achieve, 1) the closed loop poles to be a complex conjugate pair of $-0.6603 \pm \mathrm{j} 0.2463$, which corresponds to a natural frequency of $1 \mathrm{rad} / \mathrm{sec}$ and a damping ratio of $0.7,2$ ) zero steady state error to step input. It should be noted that, in contrast to classical design approaches, U-model based design procedure does not request process model (no matter line or nonlinear) in the first stage.

Consequently, for the closed loop characteristic equation, it gives

$A_{c}=R+S=q^{2}-1.3205 q+0.4966(4.4)$

where $q$ is the forward operator

To achieve the zero steady state error to a step input, it gives

$T=A_{c}(1)=1-1.3205+0.4966=0.1761(4.5)$

For the polynomials $R$ and $S$, specify

$R=q^{2}+r_{1} q+r_{2}$
$S=s_{0} q+s_{1}$

Substitute the specification of (4.6) into Diophantine to formulate the coefficients in polynomials $R$ and $S$ (Zhu and Guo 2002) with

$r_{2}+s_{1}=0.4966$
$r_{1}+s_{0}=-1.3205$

To guarantee the computation convergence of the sequence $y_{d}(t)$, that is to keep the difference equation with a stable dynamic, let $r_{1}=-0.9 \quad r_{2}=0.009$. This assignment corresponds to the characteristic equation of $y_{d}(t)$ as $(q-0.89)(q-0.01)=0$. Then the coefficients in polynomial $S$ can be determined from the Diophantine equation (Zhu and Guo 2002)

$s_{0}=-0.4205 \quad s_{1}=0.4876$

Substitute the coefficients of the polynomials $R$ and $S$ into controller of (4.3), gives rise to

$y_{d}(t+1)=0.9(t) y_{d}(t)-0.009 y_{d}(t-1)+0.1761 w(t-1)+0.4205 y(t)-0.4876 y(t-1)$

Therefore, the controller output $u(t-1)$ can be determined from resolving (3.2) by letting $y(t)=y_{d}(t)$. 
The completed control system structure is shown in Figure 4.

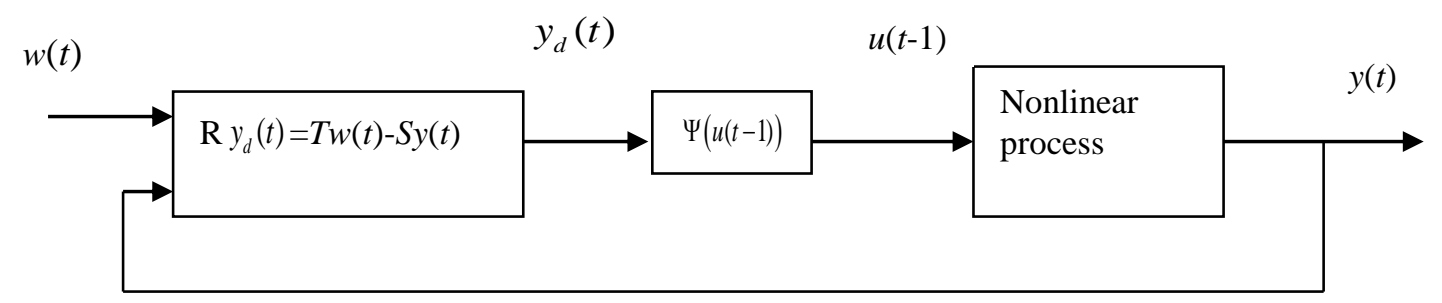

Figure 4 U-model based pole placement control system

\section{2 design of U-model based stabilised controller (U-SC)}

It should be noted that the precondition for designing a pole placement controller is that the process is stable at least within the operational region. If a process, frequently subject to nonlinear dynamics, is not globally stable or unstable in certain range of operation set points, a stabilisation controller should be considered. Accordingly, the main feature of the stabilisation control is to stabilise the process in closed loop while it works in an unstable operation interval. The stabilisation controller is designed by the following procedure.

The controller structure in this study, as described with (3.16), is specified by

Liner compensation part uses the design U-PPC

Nonlinear cancellation is designed to remove the critical nonlinear dynamic term $y^{3}(t-1)$.

The completed control system structure is shown in Figure 5.

Remark 7: It should be noted that, in general, the control design objective in such situations should not be perfect tracking or asymptotically tracking, instead it should be satisfied with, for example, bounded-error tracking, small tracking error being achieved for desired trajectories of particular interest (Slotine and Li 1991).

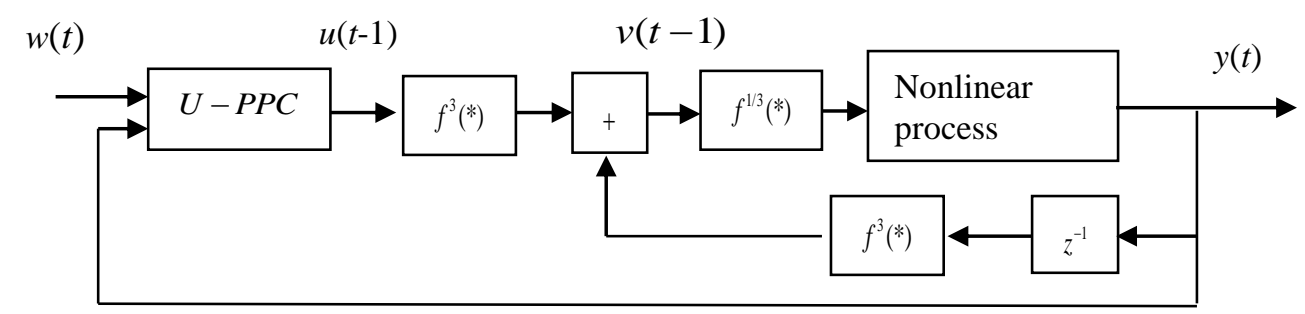

Figure 5 U-model based stabilisation control system

\section{Computational experiments --- bench tests}

This nonlinear process model of (2.3) is conditional open loop stable, which is dependent on process input amplitude. A number of computational experiments have been conducted with a set of different amplitude step input stimulations to find the model output responses are

Stable --- monotonic response (almost as a first order linear model) to a step input $|u(t)| \prec 13$

Stable --- decayed oscillatory to a step input $13 \prec|u(t)| \prec 16$

Constant oscillation to a step input $|u(t)|=16$ 
Unstable --- monotonic response to a step input $|u(t)| \succ 16$

Regarding testing of the designed control system performance, both U-PPC and U-SC are specified for controlling the temperature on the outer wall of the reaction tube in the heavy oil pyrolysis/cracking furnace, at different amplitude level of operation reference inputs.

\subsection{Bench test of U-PPC}

Assign the step reference input with amplitude between 0-5, which means the process is working within an open loop stable operation range.

The output response of the designed control systems with assigned poles and steady state properties is shown in Figure 6.1 and the pole placement controller output is shown in Figure 6.2. On inspection of the plots, it can be noted that 1) for reference temperatures, with the U-model control, the furnace temperature has been effectively regulated to follow the operational point change with the designed transient and steady state response. The decayed oscillatory response, relatively rich dynamic characteristics, was only for simulation tests, and the monotonic response can be similarly designed with the U-model approach; 2) there is a very short duration of abnormal fluctuation, within the control range, at the initial stage, which may be induced by root solver initial adaptation and/or nonlinear dynamic effect. Accordingly, the controller output gives a large reaction to supress the unwanted fluctuation. Afterwards, the furnace temperature response follows the operation reference well; 3) once again this experiment confirms that the pole placement controller is one-off designed and applicable to many different plant models, the sole difference between the applications is their U-model structure and associated parameters. If classical approaches are used, on plant changes the entire controller must be redesigned due to the dependence of the plant model in the design.

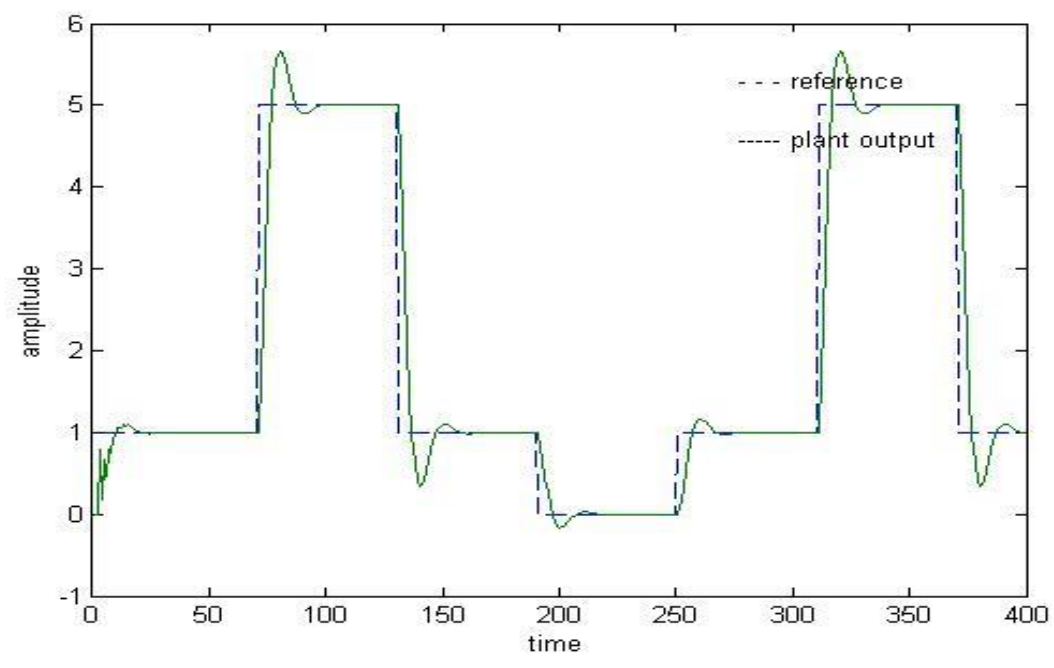

Figure 6.1 Process output response 


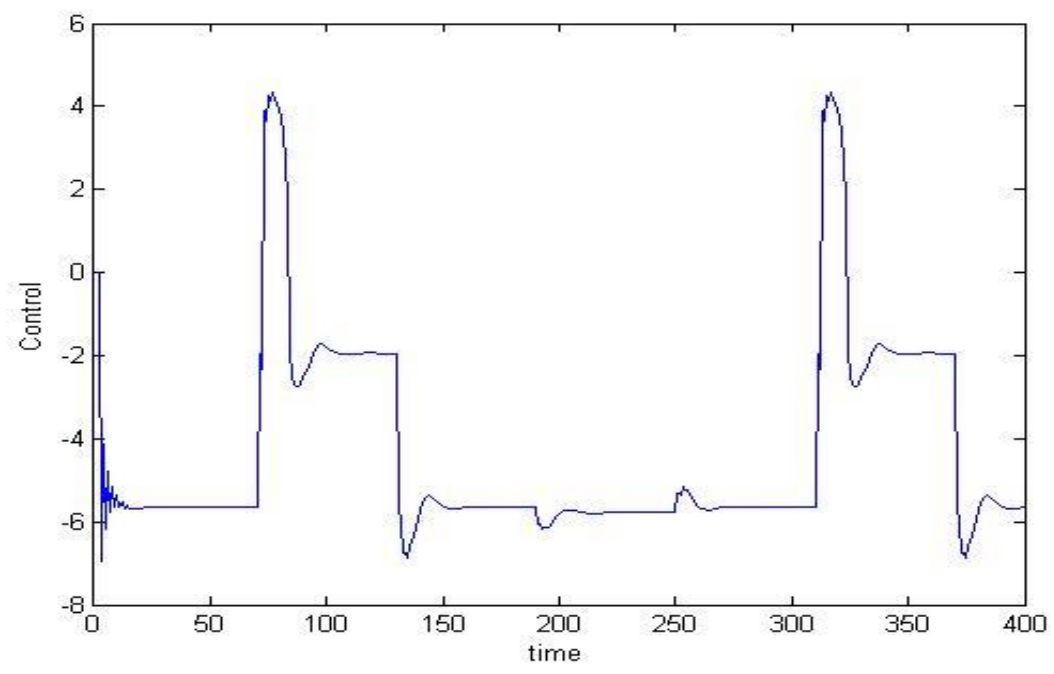

Figure 6.2 Controller output response

\subsection{Bench test of U-SC}

Assign the step reference input with amplitude between 0-30, which means that the process is working within an open loop unstable operation range.

The output response of the designed control systems with the specified error response is shown in Figure 7.1 and the controller output is shown in Figure 7.2. Inspection of the plots, the similar observation as those from Figures 6.1 and 6.2 can be concluded. Additionally, 1) the initial transient response is better, because the nonlinear term $y^{3}(t-1)$ in the plant has been cancelled by feedback stabilisation; 2) Feedback stabilisation successfully stabilises the unstable open loop process with U-model control in closed loop, with a new function added in Ucontrol toolbox; 3) controller output is reasonably good, and a large amplitude is not needed to effectively react to operational temperature variations.

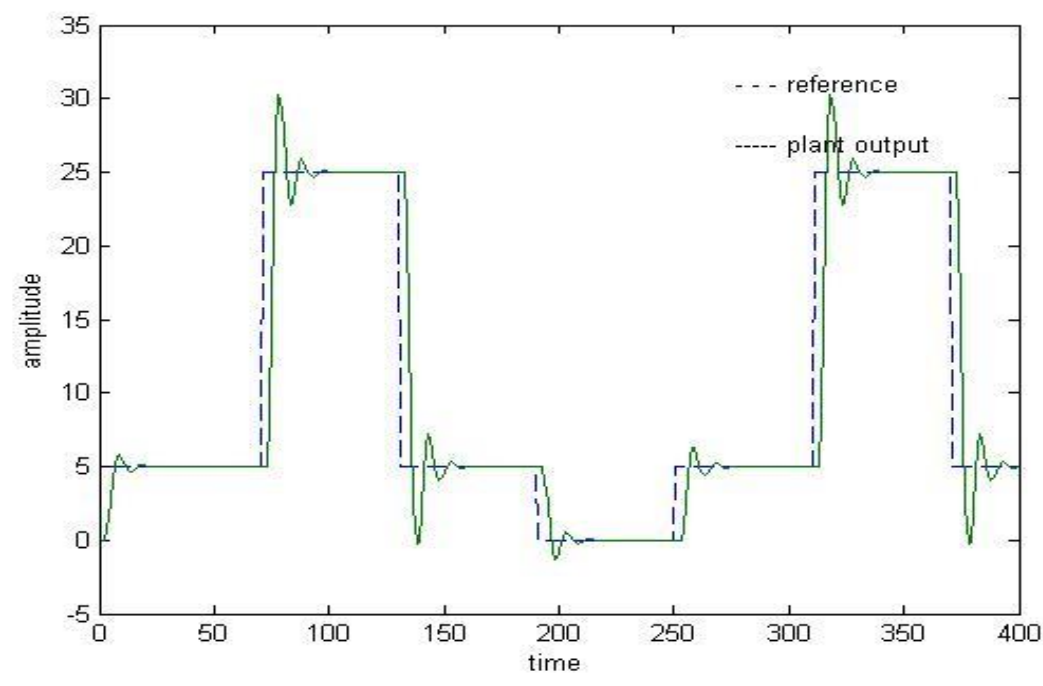

Figure 7.1 Process output response 


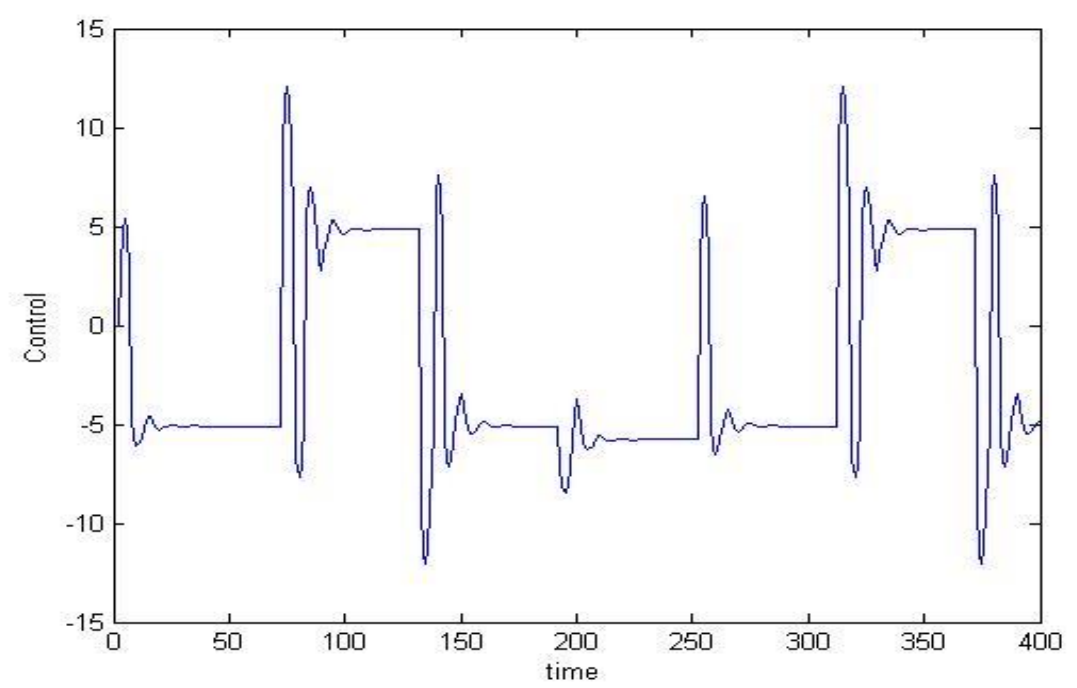

Figure 7.2 Controller output response

\section{Conclusions}

Once again this study has demonstrated the following hypothesis and research methodology claimed within the U-model framework.

1) It is possible to use linear methodologies to directly provide solutions for control of a large class of smooth nonlinear dynamic process models, and therefore to simplify and generalise nonlinear control system design by the principle of parsimony. In a critical distinction from classical approaches, U-model based design is independent of the plant/process model.

2) A three step research methodology, 1) convert a nonlinear model into a linear approach applicable structure; 2) identify the induced problems from the model conversion, 3) revise the linear approach to accommodate the identified problems in the model conversion.

Another attempt in the study is using U-model framework to design a stabilisation controller for the conditionally unstable nonlinear dynamic process. The next step will be laboratory-scale bench tests with real rigs, which may include model uncertainty, time delay, and other typical challenging issues frequently encountered in many process industries.

Last, but not least, it should be pointed out that the methodology and algorithms with U-model based control system design is relative new and little investigated, with no leading journal publications. Counter examples and comments on the weakness of the new approach are welcomed, in order to strengthen its theoretical understanding and applicability in process industries.

\section{Acknowledgements}

This work is partially supported by the National Nature Science Foundation of China under Grants 61273188 and 61473312, and Taishan Scholar Construction Engineering Special Funding, Shandong, China. The other thanks are given to Dr Steve Wright for English proof reading. Finally, the authors are grateful to the editors and the anonymous reviewers for their helpful comments and constructive suggestions with regard to the revision of the paper.

\section{References}

Apostolos, G, Ajit, V.S., and Peter, T, Ethylene optimization system reaps operations and maintenance benefits, Oil \& Gas Journal, V96, N10, 46-50, 1998.

Babik, Z. and Dostal, P., Hammerstein and Wiener Models in nonlinear control of servo-speed mechanism AMIRA DR300, Advanced Research in Scientific Areas, 1727-1733, 2012.

Billings, S.A., Nonlinear System Identification: NARMAX Methods in the time, frequency, and spatio-temporal domains, Wiley, John \& Sons, Chichester, West Sussex, 2013. 
Cheema, T.A., Ali, H., and Park, C.W., Thermal-FSI based analysis of annealing process for a steel wire in a tube furnace, Applied Thermal Engineering, V98, N5, 340-351, 2016.

Li, P., Li, Q., Lei, R., Chen, A., Ren, L., and Cao, W., Development and application of advanced process control system for ethylene cracking heaters, CIESC Journal, V62, N8, 2216-2220, 2011.

Du, W.X., Wu, X.L, and Zhu, Q.M., Direct design of U-Model based generalized predictive controller (UMGPC) for a class of nonlinear (polynomial) dynamic process, Proc. Instn. Mech. Enger, Part I: Journal of Systems and Control Engineering, V226, 27-42, 2012.

Fleliers, P. M., Reymers, G. C., and Froment, G. F., Simulation of the run length of an ethane cracking furnace, Ind Eng Chem Res, V29, 636-641, 1990.

Guo, S.C., Tube type furnace heat transfer mathematical model, Journal of Chemical Engineering, V3, 255-263, 1981.

Hasan, R., Perera, N., Baddage, and Persson, M., Numerical modelling of heat transfer in a tube furnace for steel wire annealing, 29th International Manufacturing Conference (IMC29), 20-25, University of Ulster, Northern Ireland, 29-30 August 2012.

Heynderickx, G. J., Cornelis, G. G., and Froment, G. F., Circumferential tube skin temperature profiles in thermal cracking coils, AIChE J, V38, N12, 1905-1912, 1992.

Hu, Y.F., Study on modelling of naphtha pyrolysis furnace, PhD Thesis, Tsinghua University, China, 2005.

Huang, W., Improve naphtha cracker operation, Hydrocarbon Processing, V2, 79—83, 1980.

Huang, A.Q. and Wang, Y., The Hammerstein predict model of control valve based on least square support vector machine, Applied Mechanics and Materials, V472, 164-170, 2014.

Kumar P and Kunzru D., Modeling of naphtha pyrolysis, Industrial \& Engineering Chemistry Process Design and Development, V24, N3, 774-782, 1985.

Lee, T.H., Park, J.H., Lee, S.M., and Lee, S.C., Nonlinear model predictive control for solid oxide fuel cell system based on Wiener model, International Scholarly and Scientific Research \& Innovation V4, N12, 687-692, 2010

Li, P., Li, Q., Lei, R., Chen, A., Ren, L., and Cao, W., Development and application of advanced process control system for ethylene cracking heaters, CIESC Journal, Vol62, N8, 2216-2220, 2011.

Mahmoodia, S., Poshtana, J., Jahed-Motlaghb, M.R., and Montazeria, A., Nonlinear model predictive control of a $\mathrm{pH}$ neutralization process based on Wiener-Laguerre model, Chemical Engineering Journal, V146, 328-337, 2009.

Rainara, R. M.V., Fleliers, P. M., and Froment, G. F., The coupled simulation of heat transfer and reaction in a pyrolysis furnace, Chem Eng Sci, V43, N6, 1223-1229, 1988.

Shao, H.Y., Miao, G.Y., and Zhang, Z.Q., State feedback control design for a networked control model of systems with two additive time-varying delays, Int. J. of Innovative Computing, Information and Control, V11, N4, 1457-1469, 2015.

Shi, H., Su, C. Cao, J., Li P., Liang J., and Zhong, G., Nonlinear adaptive predictive functional control based on the Takagi-Sugeno Model for average cracking outlet temperature of the Ethylene Cracking Furnace, Industrial \& Engineering Chemistry Research, V54, N6, 1849-1860, 2015.

Shi, P., Li, F.B., Wu, L.G., and Lim C.C., Neural network-based passive filtering for delayed neutral-type semiMarkovian jump systems, IEEE Trans on Neural Networks and Learning Systems, DOI: 10.1109/TNNLS.2016.2573853, 2016. 
Slotine, J.J.E. and Li, W.P., Applied nonlinear control, Prentice Hall, New Jersey, 1991

Savu, A., Lazea, G., and Agachi, P.S., Optimization and advanced control for thermal cracking processes, 20th European Symposium on Computer Aided Process Engineering - ESCAPE20, Pierucci, S. and Ferraris, G.B. (Editors), pp.20-25. 2010.

Setpoint Inc Plant optimization (Olefins) Hydrocarbon Processing, V 69 N9 pp. 90-120, 1990.

Xing, H.L., Zhong, X.Z. and Li, J., Linear extended state observer based back-stepping control for uncertain SISO nonlinear systems, Int. J. of Innovative Computing, Information and Control, V11, N4, 1411-1419, 2015, 2015.

Yang, G.J., Huang, Z.Q, and Yang, X.P., Experimental and pure radiative heat transfer model of a cylindrical tubular heater, Journal Petroleum Processing, V5, N3, 47-54, 1989.

Yu, K., Wang, X., and Wang Z., Self-adaptive multi-objective teaching-learning-based optimization and its application in ethylene cracking furnace operation optimization, Chemometrics and Intelligent Laboratory Systems, V146, N15, 198-210, 2015.

Zhang, H.T., Li, H.X., and Chen, G.R., Dual-mode predictive control algorithm for constrained Hammerstein systems, V 81, N 10, pp. 1609-1625, 2008.

Zheng, S., Zhang, X.Y., Qi, C.B., and Zhou, H.C., Modeling of heat transfer and pyrolysis reactions in ethylene cracking furnace based on 3-D combustion monitoring, International Journal of Thermal Sciences, V94, 28-36, 2015.

Zhu, Q.M., Identification and control of nonlinear systems, PhD thesis, University of Warwick, UK, 1989.

Zhu, Q.M. and Guo, L.Z., A pole placement controller for nonlinear dynamic processs, Journal of Systems and Control Engineering, Proceedings of the Institution of Mechanical Engineers Part I, V216, N6, 467-476, 2002.

Zhu, Q.M., Wang, Y.J., Zhao, D.Y., Li, S.Y., and Billings, S.A., Review of rational (total) nonlinear dynamic system modelling, identification and control, Int. J. of Systems Science, V46, N12, 122-133, 2015.

Zhu, Q.M., Zhao, D.Y. and Zhang, J.H., A general U-Block model based design procedure for nonlinear polynomial control systems, Int. J. of Systems Science, V47, N14, 3465-3475, 2016. 